\title{
Clinical effects of long-term cardiac contractility modulation (CCM) in subjects with heart failure caused by left ventricular systolic dysfunction
}

\author{
D. Müller ${ }^{1}$ (D) A. Remppis ${ }^{1} \cdot$ P. Schauerte ${ }^{2} \cdot$ S. Schmidt-Schweda ${ }^{3}$.

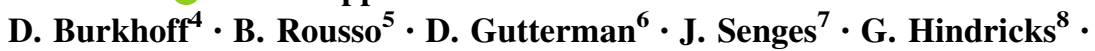 \\ K.-H. Kuck ${ }^{9}$
}

Received: 19 December 2016/ Accepted: 30 June 2017/Published online: 6 July 2017

(c) The Author(s) 2017. This article is an open access publication

\begin{abstract}
Introduction Heart failure is a major cause of morbidity and mortality throughout the world. Despite advances in therapy, nearly half of patients receiving guideline-directed medical therapy remain limited by symptoms. Cardiac contractility modulation (CCM) can improve symptoms in this population, but efficacy and safety in prospective studies has been limited to 12 months of follow-up. We report on the first 2 year multi-site evaluation of CCM in patients with heart failure.

Methods One hundred and forty-three subjects with heart failure and reduced ejection fraction were followed via clinical registry for 24 months recording NYHA class, MLWHFQ score, 6 min walk distance, LVEF, and peak $\mathrm{VO}_{2}$ at baseline and 6 month intervals as clinically indicated. Serious adverse events, and all cause as well as cardiovascular mortality were recorded. Data are presented stratified by LVEF (all subjects, LVEF $<35 \%$, LVEF $\geq 35 \%$ ).
\end{abstract}

\footnotetext{
A. Remppis

ba.remppis@hgz-bb.de

Heart and Vascular Center (HGZ), Bad Bevensen, Germany

2 University Hospital Aachen RWTH, Berlin, Germany

3 Georg August University of Gottingen, Gottingen, Germany

4 Columbia University, New York, NY, USA

5 Impulse Dynamics, Hod Hasharon, Israel

6 Medical College of Wisconsin, Milwaukee, WI, USA

7 Institut für Herzinfarktforschung, Ludwigshafen, Germany

8 Heart Center Leipzig, Leipzig, Germany

9 Asklepios Klinik St. Georg, Hamburg, Germany
}

Results One hundred and six subjects from 24 sites completed the 24 month follow-up. Baseline parameters were similar among LVEF groups. NYHA and MLWHFQ improved in all 3 groups at each time point. LVEF in the entire cohort improved 2.5, 2.9, 5.0, and 4.9\% at 6, 12, 18, and 24 months, respectively. Insufficient numbers of subjects had follow-up data for 6 min walk or peak $\mathrm{VO}_{2}$ assessment, precluding comparative analysis. Serious adverse events $(n=193)$ were observed in 91 subjects and similarly distributed between groups with LVEF $<35 \%$ and LVEF $\geq 35 \%$, and similar to other device trials for heart failure. Eighteen deaths ( 7 cardiovascularly related) over 2 years. Overall survival at 2 years was $86.4 \%$ (95\% confidence intervals: $79.3,91.2 \%$ ).

Conclusion Cardiac contractility modulation provides safe and effective long-term symptomatic and functional improvement in heart failure. These benefits were independent of baseline LVEF and were associated with a safety profile similar to published device trials.

Keywords Heart failure - CCM - Human - Clinical · Registry · Electrical stimulation · Survival · NYHA · LVEF $\cdot$ MLWHFQ

\section{Introduction}

In patients with moderate to severe chronic heart failure and reduced ejection fraction (HFrEF), the mainstay of guideline directed medical therapy (GDMT) includes use of beta-adrenergic blockers, angiotensin converting enzyme inhibitors (ACE-I) or angiotensin receptor blocking (ARB) agents, and aldosterone antagonists. Combination therapy with an $\mathrm{ARB}$ and neprolysin inhibitor (LCZ696) may be substituted for the ACE-I/ARB in 
relevant patients, and Ivabradine is indicated in select subjects with persistent sinus rates over $70 \mathrm{bpm}$. However, despite optimizing GDMT, up to $50 \%$ of patients remain symptomatic with limitation in exertional capacity, and deterioration of NYHA class, exercise endurance, and general well-being [1]. Of these, 35\% have prolonged QRS duration or LBBB and are candidates for cardiac resynchronization therapy (CRT) [2]. The remaining 65\% have a narrow $\mathrm{QRS}$ or RBBB and CRT is not less frequently indicated [3]. For these patients, cardiac contractility modulation (CCM) offers functional improvement, greater exercise tolerance, and symptomatic benefit [4-6]. Recently, CCM therapy was reviewed in the European Society of Cardiology's guidelines on acute and chronic heart failure (2016) where it was stated that "CCM may be considered in selected patients with HF" [1].

The Optimizer ${ }^{\mathrm{TM}}$ system (Impulse Dynamics, Orangeburg, NY, USA), which delivers the CCM therapy, consists of commercially available implantable leads and an externally chargeable impulse generator that delivers nonexcitatory biphasic electrical signals to two sites in the RV septum (spaced a few centimeters apart). Impulses are delivered during the absolute refractory period thereby avoiding ventricular capture. When applied this way for 5-12 h/day [4, 7, 8], CCM has been shown to elicit both pathophysiological and clinical benefits. CCM impulse delivery produces an instantaneous enhancement in contractility leading to an acute rise in LVEF over a few hours. This is associated with improved cardiac biochemistry especially in relation to cardiomyocyte calcium handling with upregulation of SERCA-2A, increased phosphorylation of phospholamban, normalization of the sodium-calcium exchanger, and a decrease in BNP [9-11]. These changes are associated with adaptive local remodeling, and a decrease in LVEDP and LVEDD which collectively drive the observed clinical improvement in patients treated with CCM. The clinical benefit includes an increase in LVEF, improved quality of life (Minnesota living with heart failure questionnaire; MLWHFQ), fewer symptoms (NYHA classification), and longer six minute walk test (6 MW), as well as an increase in peak VO [5, 7, 24]. The Optimizer ${ }^{\mathrm{TM}}$ system is compliant with available regulations and is commercially available in countries that recognize the $\mathrm{CE}$ Mark including the European Union, Russia, Brazil, India, and Australia. Despite substantial clinical experience with over 3000 implants, few reports with small numbers of subjects in specific sites have evaluated the benefit of CCM beyond one year [12-14].

The present registry was established as a means to follow patients originally enrolled in a clinical trial comparing CCM to a control group. Difficulties in recruiting matched control subjects prompted conversion to a prospective registry after 143 subjects had been implanted. The goal was to evaluate long-term (2 years) effects of CCM in each of the 143 symptomatic subjects with HFrEF including several with mid-range ejection fractions (HFmEF). Data acquisition continued until all subjects had completed baseline evaluation and follow-up at 6 months intervals for 2 years (total of 5 evaluations: baseline, 6 months, 12 months, 18 months and 24 months). These data form the basis of this prospective observational report. At baseline and at each interval the impact of CCM on NYHA, MLWHFQ, LVEF, $6 \mathrm{MW}$, and peak $\mathrm{VO}_{2}$ were recorded in accordance with data availability. Data were available at later time points only if the study was performed for clinical indications. As a result, the focus for efficacy data was on NYHA, MLWHFQ, and LVEF since follow-up measurements of $6 \mathrm{MW}$ and $\mathrm{pVO}_{2}$ were infrequently obtained. All cause mortality was also determined over the 2 year follow-up period. The present study is the first to report on longterm (2 years) effects (efficacy and safety) of CCM in HFrEF and HFmEF in a large cohort of subjects on a multi-site basis, and is the first to prospectively analyze the benefit of CCM therapy in cases with baseline LVEF below and above $35 \%$.

\section{Methods}

\section{Patient selection}

The CCM-HF investigation included 143 patients with an Optimizer device implanted for clinical heart failure and LVEF $<45 \%$ between April 15, 2010 (date of first implant) and March 25, 2015 (date of last follow-up visit). The decision to enroll subjects with $\mathrm{LVEF}>35 \%$ was based on the subgroup analysis performed on the FIX-HF-5 study $[4,7]$ which suggested that patients with LVEF between $25-45 \%$ had greater clinical benefit than those in the overall cohort. In that study $35 \%$ was used as the upper limit of baseline LVEF based on the site's evaluation but the core echo lab determined that in 38 patients, LVEF was $>35 \%$ and these subjects were analyzed separately [7]. For this reason we stratified the patients in the registry according to $\operatorname{LVEF}(<, \geq 35 \%)$, allowing us to determine if clinical effectiveness and safety of CCM were similar in subjects with baseline LVEF $\geq 35 \%$ compared to those with $\mathrm{LVEF}<35 \%$.

\section{Outcome measures}

The following efficacy data were recorded when available: NYHA classification, MLWHFQ score, ejection fraction, peak $\mathrm{VO}_{2}$, and $6 \mathrm{~min}$ walk distance (6 MW). Safety parameters were recorded including all-cause mortality 
(primary safety endpoint), cardiac mortality, and rate and severity of related serious adverse events (SAE).

Efficacy data were collected on electronic case report forms, and events were collected by the sponsor, adjudicated and reported. The main efficacy data and all safety data were monitored using an outside vendor. To minimize or avoid bias, the registry involved multiple centers (28 sites in Germany), and site selection was based upon site's experience with heart failure device implants and availability of an appropriate patient population. The incidence and nature of protocol deviations were evaluated for potential introduction of bias into the data analysis. Every effort was made to follow all subjects to assure the data set was as complete as possible.

\section{Inclusion and exclusion criteria}

Any subject over the age of 18 years who received an Optimizer system implant and provided informed consent was eligible for participation in this registry. Only those subjects who had been taking stable doses of GDMT for at least 30 days were enrolled. There were no exclusion criteria; every patient receiving an Optimizer system implant as part of the originally planned cohort study, could participate. As described above, 143 patients had CCM devices implanted at the time the study was converted to a registry. Only these patients were followed as part of the registry. All patients remained on their initial heart failure medications unless clinical circumstances required a change. There were no restrictions regarding types or doses of heart failure medications used.

\section{Study procedures and follow-up}

Initial baseline measurements included a MLWHFQ questionnaire, echocardiogram, NYHA assessment, $\mathrm{pVO}_{2}$, and a six-minute walk test.

The standard implantation protocol of the Optimizer III System used was generally followed. The precordial region of the chest (right subclavian area) was prepped and draped under sterile conditions. After access to the subclavian or cephalic vein, a lead was placed transvenously into the right atrium for sensing atrial activity. Two additional leads were placed transvenously across the tricuspid valve and secured to the right ventricular septum for sensing ventricular activity and bipolar delivery of CCM signals. After recovery from the procedure, a chest X-ray was obtained to exclude pneumothorax and to evaluate lead placement.

The Optimizer ${ }^{\mathrm{TM}}$ pulse generator was activated prior to hospital discharge for at least $2 \mathrm{~h}$, while monitoring the subject on telemetry. During this time and device parameters were adjusted as needed and at the end of $2 \mathrm{~h}$, the device was interrogated to ensure proper functioning. At the discretion of the Principal Investigator, subjects were discharged sometime after the $2 \mathrm{~h}$ monitoring, having received instructions for recharging the pulse generator including a recommendation to recharge the device weekly. Devices were programmed to be active for an average of $7 \pm 1 \mathrm{~h}$ /day. A rechargeable battery may help to match device longevity with life expectancy, a problem with most implantable devices [15].

All subjects returned for follow-up between two and four weeks after CCM activation. The pulse generator was interrogated to determine the number of sensed beats, RV lead impedances and the percent of CCM signal delivery (the number of beats actually receiving CCM relative to the total number of ventricular beats sensed during the time period when CCM was programmed to be active). Optimizer parameter settings were adjusted according to the recommendations of the site PI. The patient's ICD, if present, was also interrogated to insure absence of crosstalk with CCM.

Subjects returned to the hospital for follow-up at 6,12 , 18 and 24 months after baseline assessment. At each visit, the CCM device, and ICD if present, were interrogated to ensure proper functioning and to assess events. An interval medical history, including NYHA classification and medications was obtained. A MLWHFQ, exercise study, and a 6 min walk test were administered if clinically indicated.

At the end of the study period (24 months), the patient and site PI decided whether to maintain the Optimizer in an activated state. If signal delivery continued, follow-up visits were continued accordingly.

\section{Data validity and statistical analysis}

All efficacy data were entered by each site into a common electronic database. Adverse events were reported to the study sponsor and were adjudicated via direct communication with the investigator and reported into a separate database along with efficacy data and measurements. Categorization of serious adverse events (SAE) was done by the site PI and reviewed by the Medical Director. SAEs were categorized as arrhythmic, worsening heart failure, infectious, bleeding, ICD related, Optimizer charging issues, lead problem, death, neurological dysfunction, and renal failure. Cardiopulmonary SAEs outside the above categories were combined under the heading "general cardiopulmonary SAE", and those related to general medical events not otherwise described above were classified as "general medical SAE". Validity checks and data cleanup rules were applied with the resulting final data set used for analysis.

Our secondary analysis examined whether the clinical effects of CCM in patients with baseline LVEF $\geq 35 \%$ were no worse than (i.e., is non-inferior to) the clinical effects 
achieved by patients with initial $\mathrm{EF}<35 \%$. All data collected were analyzed comparing the follow-up interval results with baseline for the entire cohort as well as between groups, based on baseline LVEF ( $<35 \%$ vs. $\geq 35 \%$ ). Data are presented as mean $\pm \mathrm{SD}$. The significance level used was 0.05 .

In addition, analysis of the repeated longitudinal measurements was performed using mixed effects models. Models treated the time point (Baseline, 6 months, 12 months, 18 months, 24 months) as categorically fixed predictors allowing for an arbitrary average time course. Intra-subject correlation was accommodated through a subject-specific intercept and slope. The use of mixed effects models enables robust analysis, despite missing values, based on the totality of available data. In testing for improvement from baseline to follow-up, it was first tested if there is a (global) difference at any of the four follow-up times; if so then changes from baseline to specific time points are tested with allowance for multiple comparisons using Sidak's method. Comparisons between the baseline LVEF groups were made by including an interaction of the LVEF group indicator and the time variables. These computations were performed using the XTMIXED procedure in Stata 13.

\section{Ethical considerations}

The protocol was developed in accordance with the Declaration of Helsinki and ISO 14,155, and was based on the specific characteristics of the patient population under evaluation.

The study was approved by the Ethics Committee of Leipzig University (Ethik-Kommission an der Medizinischen Fakultät der Universität Leipzig, Institute for klinik pharmacology, Härtelstrasse 16-18, 04107, Leipzig, Germany) and was conducted at 28 sites in Germany.

\section{Results}

One hundred and forty-three (143) patients treated with CCM were followed in this registry. Twenty-eight subjects had baseline LVEF $\geq 35 \%$ (mean $37.3 \pm 3.1 \%$ ). All but one had an LVEF $<45 \%$. One hundred and fourteen had LVEF $<35 \%$ (mean $26.1 \pm 5.0 \%$ ) and one patient did not have a baseline LVEF recorded. This patient's data is reported in the data analysis for the entire cohort but not in the subgroup analysis by LVEF.

A total of 106 patients completed the follow-up period of 2 years in the registry. The remaining 37 either died or discontinued their participation in the study for other reasons, as detailed below. Results are presented for all 143 patients, except when noted otherwise. Of the thirty-seven
(37) patients who did not complete 24 months follow-up, nine (9) patients voluntarily withdrew their consent or were lost to follow-up, ten (10) were withdrawn due to SAE, and eighteen (18) patients died. SAEs and deaths are further discussed below.

Baseline characteristics (mean $\pm \mathrm{SD}$ ) are presented in Table 1 . When stratified by baseline LVEF $(<$ or $\geq 35 \%)$, there were no statistically significant differences between the subgroups in any baseline parameter except for the presence of an ICD and minor differences in QRS duration. Thus, the subgroups were well-matched.

Using the 3 LVEF stratifications described (EF $<35 \%$; LVEF $\geq 35 \%$; and all subjects combined), functional and quality of life (QOL) characteristics were examined at baseline and throughout the 24 months of CCM therapy.

\section{NYHA}

An improvement in NHYA was observed in overall cohort at each time point during follow-up, compared to baseline $(p<0.001)$, using a mixed effects models analysis (Sidak). A similar and statistically significant improvement in NYHA was seen in the group with LVEF $<35 \%$ and the group with LVEF $\geq 35 \%$ at each follow-up time point when compared to baseline (Table 2; Fig. 1). The mixed effects models analysis found no statistical difference in the result of the subgroups with baseline LVEF $<35 \%$ vs LVEF $\geq 35 \%$ ( $p=0.25$ for interaction).

\section{MLWHFQ}

The impact of CCM on MLWHFQ is shown in Table 2 and Fig. 1. Baseline MLWHFQ scores were similar in all three LVEF groups. The overall group improved their scores significantly at 6-months and sustained the improvement thereafter with a mean improvement of 13.9 at 6-months; 12.2 at 12 -months; 11.6 at 18 months; 12.4 at 24 -months (all $p<0.001$ ). The improvement was of similar magnitude in the two LVEF groups ( $p=0.58$ for interaction) although statistically significant improvement in MLWHFQ from baseline was observed in the LVEF $<35 \%$ and not in the LVEF $\geq 35 \%$ group on a per-time-point $t$ test, likely due to the lower number of subjects in the higher LVEF group.

\section{LVEF}

Table 2 and Fig. 2 show the changes in LVEF over the course of the study. In the overall group a statistically significant increase in ejection fraction was observed at all time points with an estimated mean improvement in LVEF of $2.5 \%$ at 6 -months, $p=0.003 ; 2.9 \%$ at 12 -months, $p=0.001 ; 5.0 \%$ at 18 -months, and $p<0.001 ; 4.9 \%$ at 
Table 1 Baseline demographics and characteristics

\begin{tabular}{|c|c|c|c|}
\hline & $\begin{array}{l}\text { All } \\
n(\%)\end{array}$ & $\begin{array}{l}\text { Group with } \mathrm{EF} \geq 35 \% \\
n(\%)\end{array}$ & $\begin{array}{l}\text { Group with EF }<35 \% \\
n(\%)\end{array}$ \\
\hline Number of patients & 143 & 28 & 114 \\
\hline Gender & $\begin{array}{l}109(76 \%) \text { Male } \\
34(24 \%) \text { Female }\end{array}$ & $\begin{array}{l}22(79 \%) \text { Male } \\
6(21 \%) \text { Female }\end{array}$ & $\begin{array}{l}87(76 \%) \text { Male } \\
27(24 \%) \text { Female }\end{array}$ \\
\hline Age [completed life years] & $62 \pm 12$ & $65 \pm 12$ & $63 \pm 12$ \\
\hline Subjects with ICD & $108(76 \%)$ & $16(57 \%)^{*}$ & $91(80 \%)$ \\
\hline Etiology of cardiomyopathy & $\begin{array}{l}69(50 \%) \text { - Ischemic } \\
57(41 \%) \text {-Idiopathic } \\
13(9 \%) \text { - other }\end{array}$ & $\begin{array}{l}N=27 \\
16(59 \%) \text {-Ischemic } \\
8(30 \%) \text {-Idiopathic } \\
3(11 \%) \text {-other }\end{array}$ & $\begin{array}{l}N=111 \\
52(47 \%) \text {-Ischemic } \\
49(44 \%) \text {-Idiopathic } \\
10(9 \%) \text {-other }\end{array}$ \\
\hline History of CABG and/or PCI & $76(57 \%)$ & $N=14(50 \%)$ & $N=61(56 \%)$ \\
\hline QRS duration (ms) & $118 \pm 26(N=131)$ & $\begin{array}{l}N=24 \\
112 \pm 17^{*}\end{array}$ & $\begin{array}{l}N=106 \\
119 \pm 27\end{array}$ \\
\hline $\begin{array}{l}\text { NYHA class } \\
{[\text { Class-N }(\%)]}\end{array}$ & $\begin{array}{l}\text { II-29 }(20 \%) \\
\text { III-103 }(72 \%) \\
\text { IV-11 }(8 \%)\end{array}$ & $\begin{array}{l}\text { II }-7(25 \%) \\
\text { III-21 }(75 \%) \\
\text { IV-0 }(0 \%)\end{array}$ & $\begin{array}{l}\text { II }-22(19 \%) \\
\text { III-81 }(71 \%) \\
\text { IV-11 (10\%) }\end{array}$ \\
\hline Hypertension-N (\%) & $66(49 \%)$ & $\begin{array}{l}N=108 \\
14(54 \%)\end{array}$ & $\begin{array}{l}N=108 \\
51(47 \%)\end{array}$ \\
\hline Presence of CRT-N (\%) & $14(10 \%)$ & $2(7 \%)$ & $12(11 \%)$ \\
\hline Cardiac medications & $N=133$ & $N=26$ & $N=107$ \\
\hline Diuretic & $104(78 \%)$ & $19(73 \%)$ & $85(79 \%)$ \\
\hline ACE-I & $82(62 \%)$ & $17(65 \%)$ & $65(61 \%)$ \\
\hline ARB & $32(24 \%)$ & $8(31 \%)$ & $24(22 \%)$ \\
\hline B-Blocker & $126(95 \%)$ & $24(92 \%)$ & $102(95 \%)$ \\
\hline Aldosterone inhibitor & $87(65 \%)$ & $18(69 \%)$ & $69(64 \%)$ \\
\hline Digoxin & $19(14 \%)$ & $4(15 \%)$ & $15(14 \%)$ \\
\hline Other medications & & & \\
\hline Anticoagulation & $49(37 \%)$ & $8(31 \%)$ & $41(38 \%)$ \\
\hline Antiplatelet Therapy & $78(59 \%)$ & $20(77 \%)$ & $58(54 \%)$ \\
\hline Statin & $92(69 \%)$ & $21(81 \%)$ & $71(66 \%)$ \\
\hline
\end{tabular}

For one subject the baseline EF was not known, hence while the entire cohort is of 143 subjects, the total number of subjects in both groups (based on baseline EF) combined, is only $142{ }^{*} p<0.05$ vs. Group with $\mathrm{EF}<35 \%$

24-months, $p<0.001$. The mixed effects model analysis found a similar improvement in LVEF at each follow-up time point between subgroups (baseline LVEF $<35 \%$ vs $\mathrm{LVEF} \geq 35 \% ; p=0.83$ for interaction.

\section{Peak $\mathrm{VO}_{2}$ and 6 min walk distance}

Only about a third of the subjects had baseline peak exercise studies performed and no more than 10 had measurements at the 12,18 and 24 month time points. Fewer than 50 subjects completed the 6 min walk distance at each follow-up time point, rendering the dataset underpowered for adequate statistical comparison.

The efficacy of medical therapy for heart failure may be influenced by the etiology of cardiac dysfunction [16] although not in all cases [17]. We examined the efficacy of
CCM in the 69 subjects with ischemic heart disease compared with those with dilated cardiomyopathy. Baseline values for NYHA $(2.9 \pm 0.5$-Isch; $2.8 \pm 0.6-\mathrm{DCM})$, MLWHFQ (46.8 $\pm 19.4-$ Isch; $45.7 \pm 17.3-$ DCM), and LVEF $\quad(29.1 \pm 6.9 \%$-Isch; $27.7 \pm 6.0$-DCM $)$ were comparable between groups. The improvement over time in each group was likewise similar (data not shown). Thus, improvement in functional and symptomatic parameters with CCM is not dependent upon whether the heart failure is idiopathic or of ischemic etiology.

Implantation of other devices during the follow-up period could have influenced clinical responses to therapy. However, very few such devices were implanted during the course of the 2 year study. Between 6 and 12 months follow-up, 1 patient received an ICD and another patient received a CRT-D. In both cases the implantation was a 
Table 2 Impact of CCM on NYHA, MLWHFQ, and LV ejection fraction over time and by EF class

\begin{tabular}{|c|c|c|c|c|c|c|}
\hline & \multirow[t]{2}{*}{ EF group } & \multirow{2}{*}{$\begin{array}{l}\text { NYHA } \\
\text { Mean }(n)\end{array}$} & \multicolumn{2}{|l|}{ MLWHFQ } & \multicolumn{2}{|c|}{ LV ejection fraction } \\
\hline & & & Value $(n)$ & $\Delta$ from baseline & $\%(n)$ & $\Delta$ from baseline \\
\hline \multirow[t]{3}{*}{ Baseline } & $\mathrm{EF}<35 \%$ & $2.9 \pm 0.5(114)$ & $45.4 \pm 19.6(104)$ & - & $26.1 \pm 5.0$ & - \\
\hline & $* \mathrm{EF} \geq 35 \%$ & $2.8 \pm 0.4(28)$ & $44.6 \pm 17.3(25)$ & - & $37.3 \pm 3.1(28)$ & - \\
\hline & Total & $2.9 \pm 0.5(143)$ & $45.0 \pm 19.2(130)$ & - & $28.3 \pm 6.4(142)$ & - \\
\hline \multirow[t]{3}{*}{6 Months } & $\mathrm{EF}<35 \%$ & $2.3 \pm 0.8^{*}(87)$ & $30.0 \pm 19.8(66)$ & $-16.4 \pm 20.8^{*}$ & $28.2 \pm 8.3(68)$ & $2.6 \pm 7.2^{*}$ \\
\hline & $\mathrm{EF} \geq 35 \%$ & $1.9 \pm 0.8^{*}(21)$ & $37.3 \pm 18.8$ & $-9.7 \pm 17.9$ & $40.5 \pm 6.2(15)$ & $3.2 \pm 6.6$ \\
\hline & Total & $2.2 \pm 0.8^{*}(109)$ & $31.4 \pm 19.7$ & $-15.1 \pm 20.3^{*}$ & $30.5 \pm 9.2(83)$ & $2.7 \pm 7.1^{*}$ \\
\hline \multirow[t]{3}{*}{12 Months } & $\mathrm{EF}<35 \%$ & $2.2 \pm 0.8^{*}(79)$ & $32.2 \pm 21.9(61)$ & $-12.3 \pm 22.8^{*}$ & $28.9 \pm 8.8(62)$ & $3.3 \pm 7.8^{*}$ \\
\hline & $\mathrm{EF} \geq 35 \%$ & $2.4 \pm 0.8^{*}(19)$ & $35.3 \pm 14.5(15)$ & $-8.9 \pm 9.9$ & $39.1 \pm 4.3(17)$ & $2.4 \pm 4.7$ \\
\hline & Total & $2.2 \pm 0.8^{*}(99)$ & $32.8 \pm 20.6(76)$ & $-11.6 \pm 20.9^{*}$ & $31.7 \pm 13.1(79)$ & $3.1 \pm 7.3^{*}$ \\
\hline \multirow[t]{3}{*}{18 Months } & $\mathrm{EF}<35 \%$ & $2.2 \pm 0.7 *(70)$ & $32.5 \pm 24.3(59)$ & $-13.0 \pm 25.6^{*}$ & $31.1 \pm 10.3$ & $5.3 \pm 9.8^{*}$ \\
\hline & $\mathrm{EF} \geq 35 \%$ & $2.1 \pm 0.6^{*}(15)$ & $35.0 \pm 16.0$ & $-4.8 \pm 15.9$ & $39.3 \pm 4.9(11)$ & $2.4 \pm 5.7$ \\
\hline & Total & $2.2 \pm 0.7 *(86)$ & $32.9 \pm 23.1(70)$ & $-11.7 \pm 24.5^{*}$ & $32.0 \pm 10.5(66)$ & $4.8 \pm 9.3^{*}$ \\
\hline \multirow[t]{3}{*}{24 Months } & $\mathrm{EF}<35 \%$ & $2.2 \pm 0.9 *(52)$ & $30.8 \pm 23.6$ & $-15.0 \pm 21.6^{*}$ & $33.0 \pm 9.1(37)$ & $7.5 \pm 9.3^{*}$ \\
\hline & $\mathrm{EF} \geq 35 \%$ & $2.3 \pm 0.7 *(15)$ & $34.5 \pm 18.7$ & $-9.4 \pm 18$ & $40.2 \pm 5.6(13)$ & $3.5 \pm 6.0$ \\
\hline & Total & $2.2 \pm 0.8^{*}(68)$ & $31.2 \pm 22.5(59)$ & $-13.6 \pm 20.6^{*}$ & $34.9 \pm 8.8(51)$ & $6.5 \pm 8.7^{*}$ \\
\hline
\end{tabular}

All data are presented as mean $\pm \mathrm{SD}$; n's reflect numbers of subjects with available data. LV ejection fraction (EF; mean $\pm \mathrm{SD})$. Means and standard deviations of available raw data are shown. $P$ values at individual time points were determined by the mixed model using Sidaks method for multiple comparisons. $* p<0.05$ vs. corresponding baseline

revision or replacement of an existing device. Two patients received a new ICD device, one between 12 and 18 months, and one between 18-24 months. All patients receiving new or revised devices were in the $\mathrm{EF}<35 \%$ group. Eliminating these patients from analysis did not change the interpretation of the results.

To determine whether improvements in functional class, quality of life, and EF might have been associated with increased use of heart failure medications (ACE-I/ARB, beta-blocker, aldosterone antagonist) we evaluated usage of these medications (initiation, termination, or maintenance) over the course of the study. Results of this analysis are shown in Table 4. The data demonstrate that few patients initiated or stopped heart failure medications over the 2 year follow-up period. Among those who did change their medical regimen, similar numbers started and stopped the medication. For each medication class (beta blockers, ACE-I/ARBs, and aldosterone antagonists), and at each time point, $80 \%$ or more of patients maintained use of the same heart failure medications that were prescribed at the baseline time point. We were not able to accurately determine changes in doses of each medication class.

\section{Serious adverse events}

Throughout the 24 months of follow-up, one hundred and ninety-three (193) serious adverse events were reported in ninety-one (91) patients (Table 3). Of these, thirty-two (32) SAEs in twenty-five (25) patients were classified by the investigator as definitely or possibly related to the device and twenty-seven (28) SAEs in twenty (20) patients as definitely or possibly related to the procedure. In view of overlap between events reported as device related and procedure related, in the aggregate there were thirty-four (34) device and/or procedure related SAEs reported in twenty-five (25) patients during the study period, most commonly due to lead migration. SAEs are presented in Table 3, stratified by baseline ejection fraction ( $<35 \%$ vs. $\geq 35 \%$ ).

Ten (10) patients were withdrawn from the study due to an SAE after a mean time of 338 days. Of these, 4 events were classified by the investigators as related or possibly related to the device and/or procedure: infection in the ICD pocket (although not in the Optimizer pocket), Optimizer IPG removal during a CRT implantation, hematoma in IPG pocket, and IPG pocket infection.

The thirty-two serious adverse events related or possibly related to the device occurred in $17 \%$ of the total study population over the study period: $17 \%$ of those with LVEF $>=35 \%$, and $18 \%$ of those with LVEF $<35 \%$. The most common of these SAEs was lead migration. During the two year period, 171 hospitalizations (all cause) occurred.

\section{Deaths}

The primary safety end-point of death of any cause occurred in 18 enrolled subjects during the 24 month follow-up period (average time from enrollment was 


\section{NHYA Classification}
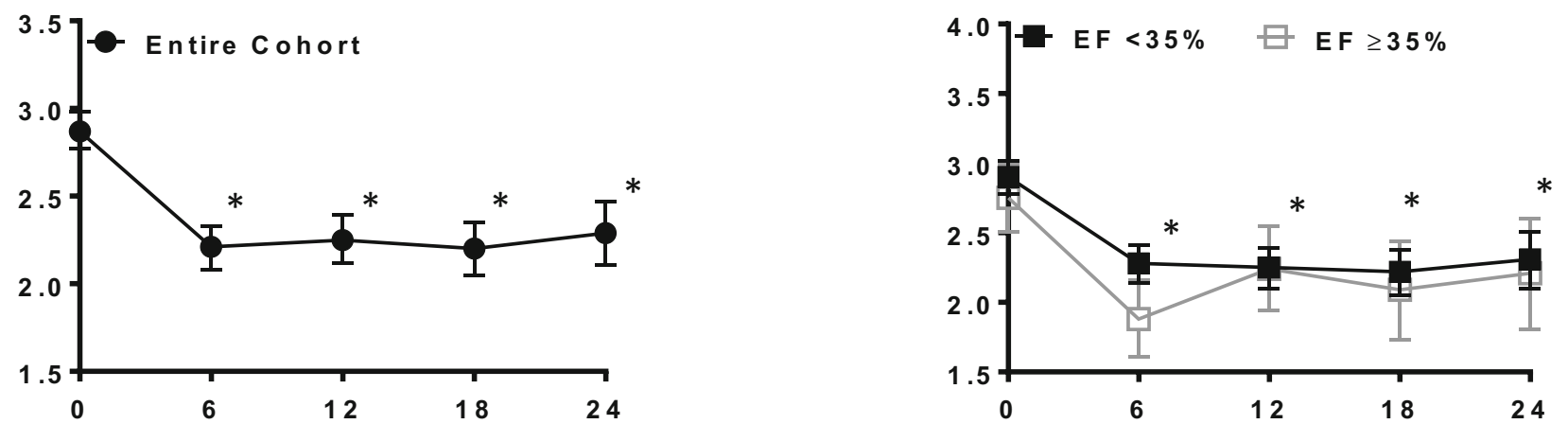

\section{MLWHFQ Score}

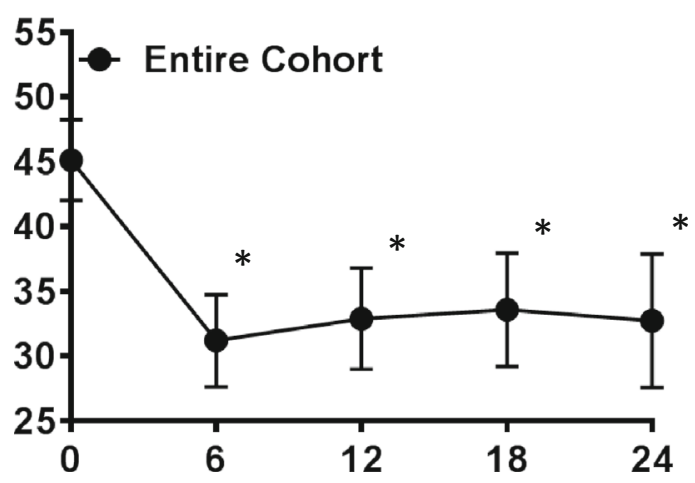

Fig. 1 Effect of CCM on NHYA and MLWHFQ. NHYA classification and MLWHFQ both showed sustained improvements over the course of the study. No difference in improvement was seen between

$341 \pm 240$ days (range 27-659 days). Three (3) of these were among the 29 patients with baseline $\mathrm{LVEF} \geq 35 \%$. Of the 18 who died, 7 deaths were classified as cardiovascular, 8 were non-cardiovascular, and in three it was not known. None of the deaths were classified by investigators as being related to the device or to the procedure. Kaplan-Meier analysis of survival estimate for all patients in this study through 2 years is shown in Fig. 2. The survival proportions and $95 \%$ CIs were $94.2 \%(88.8,97.1 \%)$ for 1 year, and $86.4 \%(79.3,91.2 \%)$ for 2 years.

\section{Discussion}

This study represents the largest long-term (24 month) efficacy and safety evaluation of heart failure patients implanted with an Optimizer device. Prior randomized trials followed patients for 3, 6 and 12 months [4, 7]. There are 2 key new findings. First, efficacy and safety of CCM are observed in patients with symptomatic heart failure for at least 2 years. Second, when patients are stratified by baseline LVEF ( $<35$ or $\geq 35 \%$ ), both groups demonstrated a similar improvement in NYHA classification, MLWHFQ

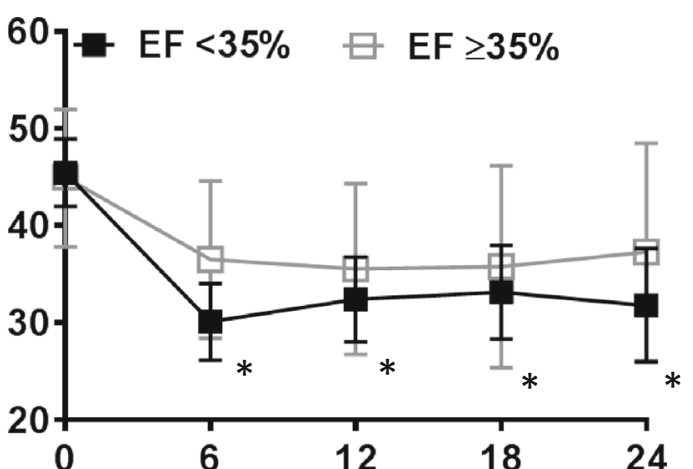

LVEF subgroups. * $p<0.05$ vs. corresponding baseline. Changes from baseline to specific time points are tested with allowance for multiple comparisons using Sidaks method mixed effects models

and LVEF at 6, 12, 18, and 24 month follow-up time points. The rates of SAEs and death were comparable between groups and consistent with prior studies.

Cardiac contractility modulation is known to be effective and safe in treating patients with chronic heart failure with ejection fractions below $35 \%$ [4, 6, 7, 18]. Secondary analysis of data from the FIX-HF-5 sub-study suggests that the efficacy of CCM is maintained and possibly greater in patients with LVEF between $25 \%$ and $45 \%$ [4]. In the present study, similar improvements in efficacy were observed when the prospectively defined analysis was stratified by baseline LVEF above and below $35 \%$.

The importance of evaluating CCM efficacy in these patients with less severely reduced ejection fractions is supported by recently published long-term mortality and hospitalization data suggesting a long-term improved survival in patients with LVEF between 25-40\% compared to those with LVEF $<25 \%$ who are treated with GDMT+CCM vs. GDMT alone [14].

NYHA symptoms improved by 0.70 points within 6 months in the entire cohort with similar changes in each subgroup. This represents a significant improvement on par with or greater than prior studies involving CCM where 


\section{A}
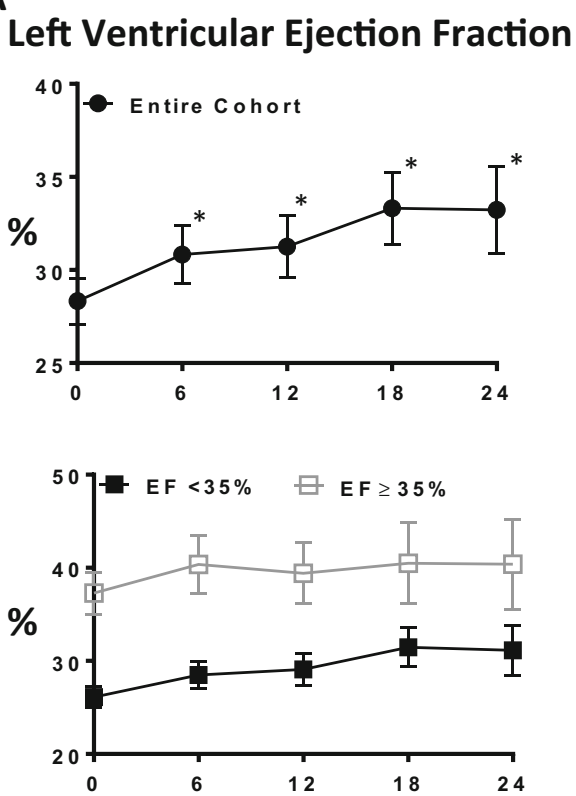

Fig. 2 Effect of $\mathrm{CCM}$ on $\mathrm{LV}$ ejection fraction and all cause mortality. a An improvement in LVEF was observed at 6 months compared to baseline and was sustained for 24 months follow-up. Improvements in LVEF were similar between LVEF subgroups. b Kaplan-Meier Survival curves for all-cause mortality over the
B

Kaplan-Meier Survival Estimate (mortality of any cause)

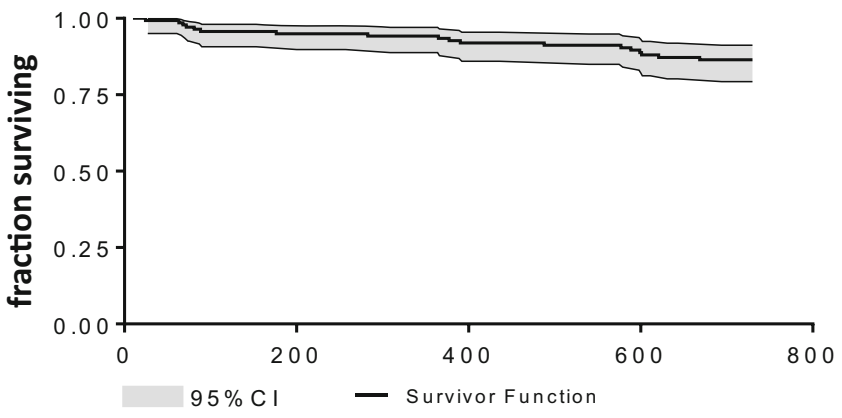

Table 3 Summary of reported Serious Adverse Events

\begin{tabular}{|c|c|c|c|c|c|c|}
\hline \multirow[t]{2}{*}{ Category } & \multicolumn{2}{|c|}{ All $(N=143)$} & \multicolumn{2}{|c|}{$\mathrm{EF} \geq 35 \%(N=29)$} & \multicolumn{2}{|c|}{$\mathrm{EF}<35 \%(N=113)$} \\
\hline & Events & Patients (\%) & Events & Patients (\%) & Events & Patients $(\%)$ \\
\hline Arrhythmia & 20 & $14(10)$ & 3 & $3(10)$ & 17 & $13(12)$ \\
\hline General cardiopulmonary & 30 & $23(16)$ & 3 & $23(10)$ & 27 & $20(17)$ \\
\hline Worsening heart failure & 55 & $37(26)$ & 11 & $6(21)$ & 44 & $33(29)$ \\
\hline Infection & 16 & $14(10)$ & 3 & $3(10)$ & 13 & $11(10)$ \\
\hline Bleeding & 5 & $4(3)$ & 1 & $1(3)$ & 4 & $3(3)$ \\
\hline ICD related & 5 & $5(3)$ & 1 & $1(3)$ & 4 & $4(4)$ \\
\hline Optimizer IPG malfunction & 5 & $5(3)$ & 2 & $2(7)$ & 3 & $3(3)$ \\
\hline Lead migration/revision & 12 & $10(7)$ & 4 & $3(10)$ & 8 & $7(6)$ \\
\hline General medical & 41 & $28(20)$ & 6 & $5(17)$ & 35 & $23(20)$ \\
\hline Death—unknown cause & 4 & $4(3)$ & - & - & 4 & $4(4)$ \\
\hline SAE probably or possibly related to device & 32 & $25(17)$ & 6 & $5(17)$ & 26 & $20(18)$ \\
\hline Total & 193 & $91(64)$ & 34 & $17(59)$ & 159 & $74(65)$ \\
\hline
\end{tabular}

Arrhythmia includes: supraventricular tachyarrhythmia (atrial fibrillation, atrial flutter, supraventricular tachycardia, ectopic atrial tachycardia), VT, and VF. General cardiopulmonary includes: angina, dyspnea, pericardial effusion/tamponade, pulmonary related (except pneumonia), syncope, venous thromboembolic disease, and valvular disease. Infection includes: ICD pocket infection, optimizer pocket infection, pneumonia, and sepsis. General medical includes: renal failure, neurological dysfunction, peripheral arterial disease/event, stroke, and other non-cardiac medical abnormalities. SAE's probably or possibly related to the device are included in the total values

subgroup stratification occurred at LVEF $=25 \%$ [19]. In the present study the markedly improved NYHA score of 0.7 was maintained throughout the 2 years of treatment.

Quality of life, assessed with the validated MLWHFQ, improved vs. baseline in the entire cohort of patients by
2 year follow-up. Data are presented as survival function together with $95 \%$ confidence limits. ${ }^{*} p<0.05$ vs. corresponding baseline. Changes from baseline to specific time points are tested with allowance for multiple comparisons using Sidaks method mixed effects models 
than those with LVEF $<25 \%$. The present registry demonstrates that although both subgroups improved over time, a trend toward greater improvement was seen in the subgroup with lower LVEF $(<35 \%)$. The reason for the differences is not clear but the small numbers of patients in the higher LVEF subgroups, study design biases, or the different comparators (randomized controls vs. withinsubject baseline values) may be explanatory factors.

Baseline LVEF among all study participants averaged $28.3 \pm 6.4 \%$ and increased at each time point studied, reaching $34.9 \pm 8.8 \%$ at 24 months. Similar and significant increases were observed in the subgroup with baseline LVEF $<35 \%$. Many fewer subjects $(n=13)$ in the group with LVEF $\geq 35 \%$ had echocardiographic assessment at 2 years follow-up, yet a strong trend toward improvement in $\mathrm{LVEF}$ was observed (LVEF $=40.2 \pm 5.6 \%, p=0.055$ vs. baseline). Lack of statistical significance likely reflects insufficient power for this parameter. The only prior randomized controlled trial that reported changes in LVEF over time had interpretable echocardiographic information in only one half of subjects randomized and saw no change in control or CCM groups over the course of the 6 month crossover trial [7].

Previous studies with small numbers of subjects have observed improvements in LVEF with relatively short-term CCM. Stix et al. examined the effect of CCM in 23 subjects with NHYA class III CHF followed for 8 weeks [18]. LVEF increased from $22 \pm 7 \%$ to $28 \pm 8 \%(p=0.0002)$ over this time. In a separate study of 13 subjects with NYHA III heart failure extending to 24 weeks, Pappone reported an improvement in LVEF during CCM from $22.7 \pm 7 \%$ to $37 \pm 13 \%(p=0.004)$ [20]. A single center long-term follow-up by Kuschyk et al [12] showed sustained improvements in LVEF, similarly to the present study. The current study is the first prospective multicenter report of sustained improvement in LVEF in patients with HFrEF and HFmEF treated with CCM. Interestingly, among the 38 patients with LVEF $<35 \%$ at baseline and who had repeat echocardiography at 24 months, 11 improved their LVEF to $>35 \%$. Six of those 11 had improved above the 35\% threshold by 6 months. This raises the question of whether CCM added to GDMT could reduce the number of patients with an indication for ICD placement.

Although trends toward improvement were observed, we found no statistically significant improvement in $6 \mathrm{MW}$ or $\mathrm{pVO}_{2}$ during follow-up, even though other prospective clinical trials did observe improvement in $6 \mathrm{MW}$ times [7, 21] at shorter follow-up times. Several factors may contribute to the lack of statistically significant improvement in $6 \mathrm{MW}$, including the small number of subjects from whom data were available (only 41 of 130 completing the study had $6 \mathrm{MW}$ testing, and 7 had $\mathrm{pVO}_{2}$ measurements at 24 months), and lack of a control group.
Similar to $6 \mathrm{MW}$, few subjects completed exercise testing throughout the study. Only 51 performed baseline exercise testing and data from 7 were available at the 24 month follow-up visit. The low participation rate likely relates to the fact that testing was done only for clinical indications since data was obtained as part of a registry. As a result it is not possible to draw any conclusions about the effects of $\mathrm{CCM}$ on $\mathrm{pVO}_{2}$ in this study. However, this question has been addressed in prior studies $[4,5,7,19]$ which demonstrate an improvement in $\mathrm{pVO}_{2}$, especially in subgroups with higher baseline LVEF [4, 6].

In our study, 14 subjects had already received a CRT device (13 with CRT-D and one with CRT-P). For most of these subjects enrollment occurred due to failure of the CRT to improve symptoms. In each case the CRT device was turned off when CCM was implanted. Although the numbers who completed follow-up functional testing are insufficient to determine efficacy of CCM in this subgroup (less than $1 / 2$ completed 24 month follow-up testing), a prior short-term study indicated that CCM can be effective in patients who fail CRT [22]. Nagele used CCM to treat 16 patients with severe heart failure who failed to respond to CRT [22]. After three months of follow-up, LVEF improved from $28.1 \pm 7 \%$ to $33 \pm 17 \%(p<0.01)$ [22].

The risk profile for CCM in this study was comparable to that described previously for patients with HFrEF and was primarily related to issues with lead malfunction. In patients with LVEF $\geq 35 \%$, SAEs were observed in $59 \%$ of patients after 2 years compared to $38 \%$ at 12 months. Similarly in patients with LVEF $<35 \%$, SAE rates were seen in $65 \%$ of subjects at 24 months and in $40 \%$ of subjects at 12 months. This is comparable to (and potentially lower than) the largest randomized controlled trial of CCM (FIX-HF-5) which reported over a 50 week period, SAEs in 61 and 54\% in the study groups [19]. Device related SAEs occurred in $13 \%$ of Optimizer treated patients in FIX-HF-5 during the 50 week follow-up, and in $17 \%$ of patients in the current study over 2 years. Mortality in the present study was similar to that observed in prior studies, although the number of deaths $(n=18$ in 2 years $)$ is too small for statistical comparison. For example in a retrospective study in 81 patients [12], Kaplan-Meier curves over a 2 year period paralleled mortality in the present study (Fig. 2). Many baseline patient characteristics were similar between the two studies (age, gender, QRS duration, NHYA class, and heart failure etiology) although LVEF was lower in the Kuschyk study $(23.1 \pm 7.9 \%)$ compared to the present study $(28.3 \pm 6.4 \%)$. Based on all the above, adverse events reported in current study reflect an acceptable safety profile consistent with prior experience using the Optimizer device and commensurate with other implantable devices in a patient population with similar acuity. 
Malignant arrhythmia generation is of particular concern in heart failure since this accounts for a large percentage of deaths. Implantation of ICDs has improved survival in this regard. The precise effect of CCM on ventricular arrhythmias has not been directly studied. It is known that application of current, sub-threshold for ventricular capture, applied to the heart during the refractory period can reduce or terminate ventricular tachycardia [23]. Whether CCM evokes similar protection has not been systematically studied although substudy analysis of one clinical trial suggests that CCM has no effect on PVCs or duration of VT [24]. In another report by Pappone et al. [20] of 13 patients followed for 8 weeks, CCM was associated with fewer daily episodes of NSVT and a trend toward a reduction in PVCs. In the largest randomized prospective clinical trial of CCM in heart failure, no increase in ventricular arrhythmias or discharge of ICDs was observed [19]. For patients with an indication for CCM and with symptomatic PVCs, it will be interesting to see if CCM might avert the need for PVC ablation [25]. It would also be of value to examine structural characteristics of the failing heart that might identify super responders, as has been done for CRT [26].

\section{Study limitations}

There are several potential limitations to this study. Significant improvement from baseline over the time course of this study was observed in several subjective metrics related to quality of life and symptoms. We believe these changes to be valid and not substantively influenced by the prominent placebo effect common with device therapy since prior studies involving CCM showed that the initial placebo effect was not sustained beyond 3 months [7] (FIX-HF-4). An objective outcome measure, ejection fraction also improved in both the subgroup with LVEF $<35 \%$ and in the total cohort at each of the follow-up time points. This further argues against a prominent placebo effect, and supports real and sustainable benefit of CCM therapy.

In a registry, follow-up testing is performed based on clinical need. This factor limited the number of patients available with outcomes data related to LVEF and exercise tolerance including 6 min walk test, and peak $\mathrm{VO}_{2}$. Nevertheless, we were able to reliably measure NYHA and MLWHFQ in a large number of subjects through the entire 2 year follow-up period.

Improvement in NYHA, MLWHFQ, and LVEF could have resulted from the increased use of pharmacological treatment of heart failure in these patients. However, analysis of use of heart failure medications (Table 4) reveals that very few subjects initiated or terminated heart failure medicine use over the course of the study. A similar and small number of patients started and stopped specific medications with the majority $(>80 \%)$ maintaining the same regimen used at enrollment. This analysis suggests that additional medical therapy is not likely the explanation for improvement in measured parameters over the time course of this study. We cannot exclude a change in dosage of heart failure medications as contributing to improved

Table 4 Variation in medication use by subjects over the course of the study

\begin{tabular}{|c|c|c|c|c|c|}
\hline & \multirow[t]{2}{*}{ Patients Chronically Treated at Baseline (\%) } & \multicolumn{4}{|c|}{ Patient numbers ( $\%$ of reported data) } \\
\hline & & Base- 6 mo & Base-12 mo & Base-18 mo & Base-24 mo \\
\hline ACE-I/ARB & $112(84)$ & & & & \\
\hline Added & & $3(3)$ & $4(4)$ & $6(7)$ & $6(8)$ \\
\hline Stopped & & $6(6)$ & $7(7)$ & $7(8)$ & $7(9)$ \\
\hline No change or not used & & $97(92)$ & $93(89)$ & $80(86)$ & $62(83)$ \\
\hline \# of patients with data & 134 & 106 & 105 & 95 & 77 \\
\hline Beta-blocker & $127(95)$ & & & & \\
\hline Added & & $1(1)$ & $(2)$ & $2(2)$ & $2(3)$ \\
\hline Stopped & & $3(3)$ & $3(2)$ & $4(4)$ & $4(5)$ \\
\hline No change or not used & & $102(96)$ & $99(95)$ & $87(94)$ & $69(92)$ \\
\hline Aldosterone antagonist & $87(65)$ & & & & \\
\hline Added & & $7(7)$ & $9(9)$ & $7(8)$ & $6(8)$ \\
\hline Stopped & & $8(7)$ & $12(11)$ & $11(12)$ & $7(9)$ \\
\hline No change or not used & & $91(86)$ & $83(80)$ & $75(80)$ & $62(83)$ \\
\hline
\end{tabular}

The number of patients with reported data at each timepoint is shown (\# of patients with data). The number of patients where drug was added, stopped, or unchanged/not used is shown in columns on the right. There was no difference in medication use from baseline to any of three time points $(6,12,18$, or 24 months). This was true for all three classes of heart failure medications (ACE-I/ARB, beta-blockers, aldosterone antagonists) 
outcomes, but, inclusion criteria required stable use of guideline-directed heart failure therapy for one month prior to enrollment, thus optimal doses were likely already achieved at the beginning of the study.

Lack of a control group poses limitations on interpretation of findings. Contemporary and comparable controls provide rigor in study design to help avoid interference from unrelated and/or unknown sources that could influence the outcome measures. Without a control group, the analysis of this study compared changes over time to baseline measurements. While this controls for inter-patient variability, it may create bias against the CCM intervention since implicit in the analysis it is assumed that baseline function remains constant over time in untreated patients. In fact, outcome variables tend to get worse over time on GDMT, thus the present study might have underestimated the benefit of CCM in this population.

Future studies should identify key biomarkers to predict optimum responsiveness to CCM. Presence of CheyneStokes respiratory patterns [27], CRP, angiopoetin [28] and other serum markers should be examined in relation to CCM efficacy.

\section{Summary}

In summary, in patients with heart failure with reduced LVEF and persistent symptoms despite GDMT, CCM provides sustained improvement in both cardiac function and QOL. The benefit is present not only in subjects with baseline LVEF $<35 \%$, but is also in those with LVEF $\geq 35 \%$. The extended benefit is not associated with an adverse impact on safety beyond what is expected with implantable devices. Consistent with previous clinical studies, these data suggest that CCM may be beneficial in select patients with heart failure, narrow QRS, and symptoms despite optimal medical management.

\section{Compliance with ethical standards}

Disclosures Rousso is employed by Impulse Dynamics, Burkhoff and Gutterman are consults for Impulse Dynamics. Support for this study was provided by Impulse Dynamics.

Informed consent All subject provided informed consent. Participating investigators and associated clinical research sites in Germany include: C Restle, C Menz, J Stockinger-Bad Krozingen; Sperzel J, Bruder O, Blank E, Waidelich L, Keinhorst J, Reuter V, Schmitz D, Steffen M-Essen; Frommhold M, Meiland R, Wagner A-Bad Berka; Muller S, Schmidt-Schweda S-Worbis; Schmidt T, Scholl C, Obergfoll M-Heilbronn; Bucholz M, Gebhardt S, Spencker S, Atmowihardjo I, Forster S, Szczesnlak S, Stoeckicht Y, Huseyin IBerlin; Reith S, Zink M, Heuer G-Aachen; Hohn A, Schwartzmann L, Hornlein C, Schertel-Grunler B, Brachmann J, Denninger PCoburg; Siems M,Latzko C, Müller D, Nickling E-Bad Bevensen; Maroto Y Jarvinen S, Block M, von Bodman G-Munchen; Beauport J, Hofmann W, Antz M-Oldenburg; Zander-Wiegmann M,
Bittlinsky A, Prull M-Herne; Andreas K, Przibille O-Frankfurt; Frohlich-Grimm F, Danschel W-Chemnitz; Aydin A, Wilke I, Schnapp A-Reinbek; Haacke K, Gunther M-Dresden; Mletzko $\mathrm{R}$-Hamburg; Karosiene Z, Bernard L, Willms-Weirich N-Ludenscheid; Brilla K, Minden H-Hennigsdorf; Over M, Hugl B, Findeisen Z, Haufe A, Wessling P-Neuwied.

Open Access This article is distributed under the terms of the Creative Commons Attribution 4.0 International License (http://crea tivecommons.org/licenses/by/4.0/), which permits unrestricted use, distribution, and reproduction in any medium, provided you give appropriate credit to the original author(s) and the source, provide a link to the Creative Commons license, and indicate if changes were made.

\section{References}

1. Ponikowski P, Voors AA, Anker SD, Bueno H, Cleland JG, Coats AJ, Falk V, Gonzalez-Juanatey JR, Harjola VP, Jankowska EA, Jessup M, Linde C, Nihoyannopoulos P, Parissis JT, Pieske B, Riley JP, Rosano GM, Ruilope LM, Ruschitzka F, Rutten FH, van der Meer P, Authors/Task Force M and Document R (2016) ESC guidelines for the diagnosis and treatment of acute and chronic heart failure: the task force for the diagnosis and treatment of acute and chronic heart failure of the European Society of Cardiology (ESC) Developed with the special contribution of the Heart Failure Association (HFA) of the ESC. Eur Heart J 18(8):891-975

2. Lund LH, Jurga J, Edner M, Benson L, Dahlstrom U, Linde C, Alehagen U (2013) Prevalence, correlates, and prognostic significance of QRS prolongation in heart failure with reduced and preserved ejection fraction. Eur Heart J 34:529-539

3. Stockburger M, Moss AJ, Klein HU, Zareba W, Goldenberg I, Biton Y, McNitt S, Kutyifa V (2016) Sustained clinical benefit of cardiac resynchronization therapy in non-LBBB patients with prolonged PR-interval: MADIT-CRT long-term follow-up. Clin Res Cardiol 105:944-952

4. Abraham WT, Nademanee K, Volosin K, Krueger S, Neelagaru S, Raval N, Obel O, Weiner S, Wish M, Carson P, Ellenbogen K, Bourge R, Parides M, Chiacchierini RP, Goldsmith R, Goldstein S, Mika Y, Burkhoff D, Kadish A, Investigators F-H- and Coordinators (2011) Subgroup analysis of a randomized controlled trial evaluating the safety and efficacy of cardiac contractility modulation in advanced heart failure. J Card Fail 17:710-717

5. Giallauria F, Vigorito C, Piepoli MF, Stewart Coats AJ (2014) Effects of cardiac contractility modulation by non-excitatory electrical stimulation on exercise capacity and quality of life: an individual patient's data meta-analysis of randomized controlled trials. Int J Cardiol 175:352-357

6. Borggrefe M, Burkhoff D (2012) Clinical effects of cardiac contractility modulation (CCM) as a treatment for chronic heart failure. Eur J Heart Fail 14:703-712

7. Borggrefe MM, Lawo T, Butter C, Schmidinger H, Lunati M, Pieske B, Misier AR, Curnis A, Bocker D, Remppis A, Kautzner J, Stuhlinger M, Leclerq C, Taborsky M, Frigerio M, Parides M, Burkhoff D, Hindricks G (2008) Randomized, double blind study of non-excitatory, cardiac contractility modulation electrical impulses for symptomatic heart failure. Eur Heart J 29:1019-1028

8. Kloppe A, Mijic D, Schiedat F, Bogossian H, Mugge A, Rousso B, Lemke B (2016) A randomized comparison of 5 versus 12 hours per day of cardiac contractility modulation treatment for heart failure patients: a preliminary report. Cardiol J. 23:114-119 
9. Gupta RC, Mishra S, Rastogi S, Wang M, Rousso B, Mika Y, Remppis A, Sabbah HN (2009) $\mathrm{Ca}(2+)$-binding proteins in dogs with heart failure: effects of cardiac contractility modulation electrical signals. Clin Transl Sci 2:211-215

10. Butter C, Rastogi S, Minden HH, Meyhofer J, Burkhoff D, Sabbah HN (2008) Cardiac contractility modulation electrical signals improve myocardial gene expression in patients with heart failure. J Am Coll Cardiol 51:1784-1789

11. Lyon AR, Samara MA, Feldman DS (2013) Cardiac contractility modulation therapy in advanced systolic heart failure. Nature Rev Cardiol 10:584-598

12. Kuschyk J, Roeger S, Schneider R, Streitner F, Stach K, Rudic B, Weiss C, Schimpf R, Papavasilliu T, Rousso B, Burkhoff D, Borggrefe M (2015) Efficacy and survival in patients with cardiac contractility modulation: long-term single center experience in 81 patients. Int J Cardiol 183:76-81

13. Kloppe A, Lawo T, Mijic D, Schiedat F, Muegge A, Lemke B (2016) Long-term survival with cardiac contractility modulation in patients with NYHA II or III. Int J Cardiol 209:291-295

14. Liu M, Fang F, Luo XX, Shlomo BH, Burkhoff D, Chan JY, Chan CP, Cheung L, Rousso B, Gutterman D, Yu CM (2016) Improvement of long-term survival by cardiac contractility modulation in heart failure patients: a case-control study. Int $\mathbf{J}$ Cardiol 206:122-126

15. Neuzner $\mathbf{J}$ (2015) The mismatch between patient life expectancy and the service life of implantable devices in current cardioverterdefibrillator therapy: a call for larger device batteries. Clin Res Cardiol 104:456-460

16. McLeod CJ, Shen WK, Rea RF, Friedman PA, Hayes DL, Wokhlu A, Webster TL, Wiste HJ, Hodge DO, Bradley DJ, Hammill SC, Packer DL, Cha YM (2011) Differential outcome of cardiac resynchronization therapy in ischemic cardiomyopathy and idiopathic dilated cardiomyopathy. Heart Rhythm: Off $\mathbf{J}$ Heart Rhythm Soc 8:377-382

17. Streitner F, Kuschyk J, Dietrich C, Mahl E, Streitner I, Doesch C, Veltmann C, Schimpf R, Wolpert C, Borggrefe M (2011) Comparison of ventricular tachyarrhythmia characteristics in patients with idiopathic dilated or ischemic cardiomyopathy and defibrillators implanted for primary prevention. Clin Cardiol 34:604-609

18. Stix G, Borggrefe M, Wolpert C, Hindricks G, Kottkamp H, Bocker D, Wichter T, Mika Y, Ben-Haim S, Burkhoff D, Wolzt M, Schmidinger H (2004) Chronic electrical stimulation during the absolute refractory period of the myocardium improves severe heart failure. Eur Heart J 25:650-655

19. Kadish A, Nademanee K, Volosin K, Krueger S, Neelagaru S, Raval N, Obel O, Weiner S, Wish M, Carson P, Ellenbogen K, Bourge R, Parides M, Chiacchierini RP, Goldsmith R, Goldstein S, Mika Y, Burkhoff D, Abraham WT (2011) A randomized controlled trial evaluating the safety and efficacy of cardiac contractility modulation in advanced heart failure. Am Heart J 161(329-337):e1-e2

20. Pappone C, Augello G, Rosanio S, Vicedomini G, Santinelli V, Romano M, Agricola E, Maggi F, Buchmayr G, Moretti G, Mika Y, Ben-Haim SA, Wolzt M, Stix G, Schmidinger H (2004) First human chronic experience with cardiac contractility modulation by nonexcitatory electrical currents for treating systolic heart failure: mid-term safety and efficacy results from a multicenter study. J Cardiovasc Electrophysiol 15:418-427

21. Neelagaru SB, Sanchez JE, Lau SK, Greenberg SM, Raval NY, Worley S, Kalman J, Merliss AD, Krueger S, Wood M, Wish M, Burkhoff D, Nademanee K (2006) Nonexcitatory, cardiac contractility modulation electrical impulses: feasibility study for advanced heart failure in patients with normal QRS duration. Heart rhythm: Off J Heart Rhythm Soc 3:1140-1147

22. Nagele H, Behrens S, Eisermann C (2008) Cardiac contractility modulation in non-responders to cardiac resynchronization therapy. Eur: Eur Pacing, Arrhythm Card Electrophysiol: J Work Groups Card Pacing, Arrhythm, Card Cell Electrophysiol Eur Soc Cardiol 10:1375-1380

23. Salama G, Kanai A, Efimov IR (1994) Subthreshold stimulation of Purkinje fibers interrupts ventricular tachycardia in intact hearts. Experimental study with voltage-sensitive dyes and imaging techniques. Circ Res 74:604-619

24. Lawo T, Borggrefe M, Butter C, Hindricks G, Schmidinger H, Mika Y, Burkhoff D, Pappone C, Sabbah HN (2005) Electrical signals applied during the absolute refractory period: an investigational treatment for advanced heart failure in patients with normal QRS duration. J Am Coll Cardiol 46:2229-2236

25. Fichtner S, Senges J, Hochadel M, Tilz R, Willems S, Eckardt L, Deneke T, Lewalter T, Dorwarth U, Reithmann C, Brachmann J, Steinbeck G, Kaab S, German Ablation R (2017) Safety and efficacy in ablation of premature ventricular contraction: data from the German ablation registry. Clin Res Cardiol 106:49-57

26. Hurlimann D, Schmidt S, Seifert B, Saguner AM, Hindricks G, Luscher TF, Ruschitzka F, Steffel J (2015) Outcome of superresponders to cardiac resynchronization therapy defined by endpoint-derived parameters of left ventricular remodeling: a twocenter retrospective study. Clin Res Cardiol 104:136-144

27. Oldenburg O, Spiesshofer J, Fox H, Bitter T, Horstkotte D (2015) Cheyne-Stokes respiration in heart failure: friend or foe? Hemodynamic effects of hyperventilation in heart failure patients and healthy volunteers. Clin Res Cardiol 104:328-333

28. Poss J, Ukena C, Kindermann I, Ehrlich P, Fuernau G, Ewen S, Mahfoud F, Kriechbaum S, Bohm M, Link A (2015) Angiopoietin-2 and outcome in patients with acute decompensated heart failure. Clin Res Cardiol 104:380-387 